\title{
Clinical Outcome and Mechanisms of Deep Brain Stimulation for Obsessive-Compulsive Disorder
}

\author{
Maarten van Westen • Erik Rietveld • Martijn Figee • \\ Damiaan Denys
}

Published online: 11 March 2015

(C) The Author(s) 2015. This article is published with open access at Springerlink.com

\begin{abstract}
Clinical outcome of deep brain stimulation (DBS) for obsessive-compulsive disorder (OCD) shows robust effects in terms of a mean Yale-Brown Obsessive-Compulsive Scale (YBOCS) reduction of $47.7 \%$ and a mean response percentage (minimum $35 \%$ YBOCS reduction) of $58.2 \%$. It appears that most patients regain a normal quality of life (QoL) after DBS. Reviewing the literature of the last 4 years, we argue that the mechanisms of action of DBS are a combination of excitatory and inhibitory as well as local and distal effects. Evidence from DBS animal models converges with human DBS EEG and imaging findings, in that DBS may be effective for OCD by reduction of hyperconnectivity
\end{abstract}

This article is part of the Topical Collection on Neuromodulation

M. van Westen $(\bowtie) \cdot$ E. Rietveld $\cdot$ M. Figee $\cdot$ D. Denys

Department of Psychiatry, Academic Medical Center,

Meibergdreef 9, 1105 AZ Amsterdam, The Netherlands

e-mail: m.vanwesten@amc.nl

E. Rietveld

e-mail: d.w.rietveld@amc.nl

M. Figee

e-mail: m.figee@amc.nl

D. Denys

e-mail: ddenys@gmail.com

E. Rietveld $\cdot$ M. Figee $\cdot$ D. Denys

Amsterdam Brain and Cognition Center, University of Amsterdam,

Nieuwe Achtergracht 129 (Building L), 1018

WS Amsterdam, The Netherlands

D. Denys

The Netherlands Institute for Neuroscience, Royal Netherlands

Academy of Arts and Sciences, Meibergdreef 47, 1105

BA Amsterdam, The Netherlands

\section{E. Rietveld}

Department of Philosophy, Institute for Logic, Language and

Computation, University of Amsterdam, Science Park 107,

1098 XG Amsterdam, The Netherlands between frontal and striatal areas. This is achieved through reduction of top-down-directed synchrony and reduction of frontal low-frequency oscillations. DBS appears to counteract striatal dysfunction through an increase in striatal dopamine and through improvement of reward processing. DBS affects anxiety levels through reduction of stress hormones and improvement of fear extinction.

Keywords Deep brain stimulation - Obsessive-compulsive disorder $\cdot$ Clinical outcome $\cdot$ Quality of life $\cdot$ Mechanisms of action

\section{Introduction}

Obsessive-compulsive disorder (OCD) affects $2 \%$ of the general population [1], causing severe functional impairment as a result of anxiety, persistent intrusive thoughts (obsessions), and behavioral inflexibility (compulsions). A small fraction of these patients is unresponsive to standard treatment regimes with cognitive behavioral therapy (CBT), multiple serotonin reuptake inhibitors (SRIs), clomipramine, and addition of antipsychotics [2]. For a subset of these treatment refractory patients, ablative and neuromodulatory strategies are the last options. Recently, deep brain stimulation (DBS) has been approved as a reversible alternative for ablative stereotactic neurosurgery or gamma-knife radiotherapy [3••]. DBS is a chronic and invasive neuromodulatory technique with the unique possibility of fine tuning its effects. DBS involves stereotactic implantation of electrodes in deep brain structures [4], followed by a trial and error process of optimizing parameter settings through telemetry in response to the patient's feedback and clinical scores. 
This article critically reviews recent evidence on DBS for OCD in order to bring the reader up to date with respect to (1) clinical outcome in terms of Yale-Brown Obsessive-Compulsive Scale (YBOCS) and quality of life (QoL) and (2) recent (January 2011-September 2014) clinical and preclinical evidence on mechanisms of action of DBS. We will discuss side effects elsewhere. We conducted an electronic literature search covering journals with indexations in Embase, PsycINFO, and PubMed as well as the Cochrane Library and the WHO trial register, using the text words "obsessive-compulsive disorder" and "deep brain stimulation." Because this combination yielded a limited amount of hits and using more specific search terms would introduce more selection bias, we decided to manually select relevant studies. All primary clinical outcome studies as well as case reports were included. All clinical and preclinical studies on mechanisms of action from 2011 onward were included. We selectively included articles before 2011 in so far as they helped to sketch the background for our discussion of the recent studies.

\section{Clinical Outcome}

We identified 23 trials [5-27], and case reports that assessed clinical outcome in terms of YBOCS scores of DBS for OCD were included (see Table 1). It concerns 108 uniquely implanted patients, of which 49 were included in the studies of Mallet et al. [22], Denys et al. [19], Goodman et al. [8], and Huff et al. [18] that used a double-blind sham-controlled crossover design. Five targets were used, including white matter tracts in the anterior limb of the internal capsule (ALIC) or ventral capsule (VC) and the inferior thalamic peduncle (ITP) and gray matter structures in the ventral striatum (VS), the nucleus accumbens (NAc), and the subthalamic nucleus (STN) [28]. Follow-up varied between 6 and 36 months.

Clinical outcome for all studies, together with each uniquely implanted patient weighted once, yields a mean YBOCS reduction of $47.7 \%$ and of $37.4 \%$ for only the four randomized sham-controlled trials (RCTs). The mean response percentage (minimum $35 \%$ YBOCS reduction [2]) is $58.2 \%$ for all studies and $43.8 \%$ for the double-blind sham-controlled studies, leaving out the study of Mallet et al. that used a lower cutoff point of $25 \%$ [22]. Placebo effects appear to be minimal, as three out of four RCTs demonstrate significant difference between sham and stimulation conditions. VC/VS and bilateral NAc targets, although more extensively studied, show greater symptom improvement than the STN, and bilateral NAc stimulation is more effective than right unilateral NAc stimulation.

One presumed meta-analysis [29] miscalculated effects and had considerable inclusion bias [30]. We argue that differences in the designs of available studies currently obstruct the conduction of a meta-analysis. Compatibility might be increased through consistent use and publication of end points and response rates. The same goes for standardization of parameter setting optimization of which the additional effect is difficult to apprehend. One should also note that the high prevalence of comorbid major depressive disorder (MDD) may mediate effects on OCD. Lastly, more RCTs as well as comparative studies between various targets, especially between VC/VS and STN, are necessary.

\section{Quality of Life}

Half of the DBS studies assessed Global Assessment of Functioning (GAF) scores as a measure of QoL, which amounts to a mean DBS-related GAF increase of 21.5 out of 100 points. Interestingly, the study of Huff et al. [18] had a similar mean GAF improvement despite the fact that only one of these patients was a responder in terms of YBOCS, suggesting that DBS may improve QoL independent of symptom improvement. Correspondingly, the first clinical study on long-term QoL effects of DBS for OCD found significant improvement on the 26-item WHO QoL Scale-Brief Version (WHOQOLBREF), even in nonresponders in terms of YBOCS [31]. At baseline, DBS-eligible OCD patients were impaired in all five domains compared with age- and sex-matched healthy controls. The physical and psychological domains, which were most severely impaired at baseline, improved with 23 and $27 \%$, respectively, at 8 months post-surgery, and with a total of $39.5 \%$ at $3-5$ years post-surgery compared to baseline. Improvement in physical and environmental domains correlated with symptom improvement on the YBOCS and with improvement of depression and anxiety on the Hamilton Rating Scale for Depression (HAM-D) and Hamilton Rating Scale for Anxiety (HAM-A). The environmental domain (financial, housing, mobility) also improved significantly with $16 \%$. Only the social domain did not significantly improve, and this was especially due to patients with long illness durations. The overall score improved with $90 \%$, approaching the normal range, indicating that patients might in fact regain a normal standard of QoL. It appears, however, that DBS is a chronic treatment and that improvements on QoL disappear after acute DBS cessation. This also causes a relapse of obsessions and compulsions and a rebound of anxiety and depression which exceeds pre-surgery levels with approximately $40 \%$ [32].

\section{Mechanisms of Action}

The mechanisms of action of DBS that mediate the clinical effects discussed above were initially understood as equivalent to the lesion effects of ablative surgery [6]. DBS was thought to locally inhibit excessive pathological activity. In recent years, it has been acknowledged that facilitation of 


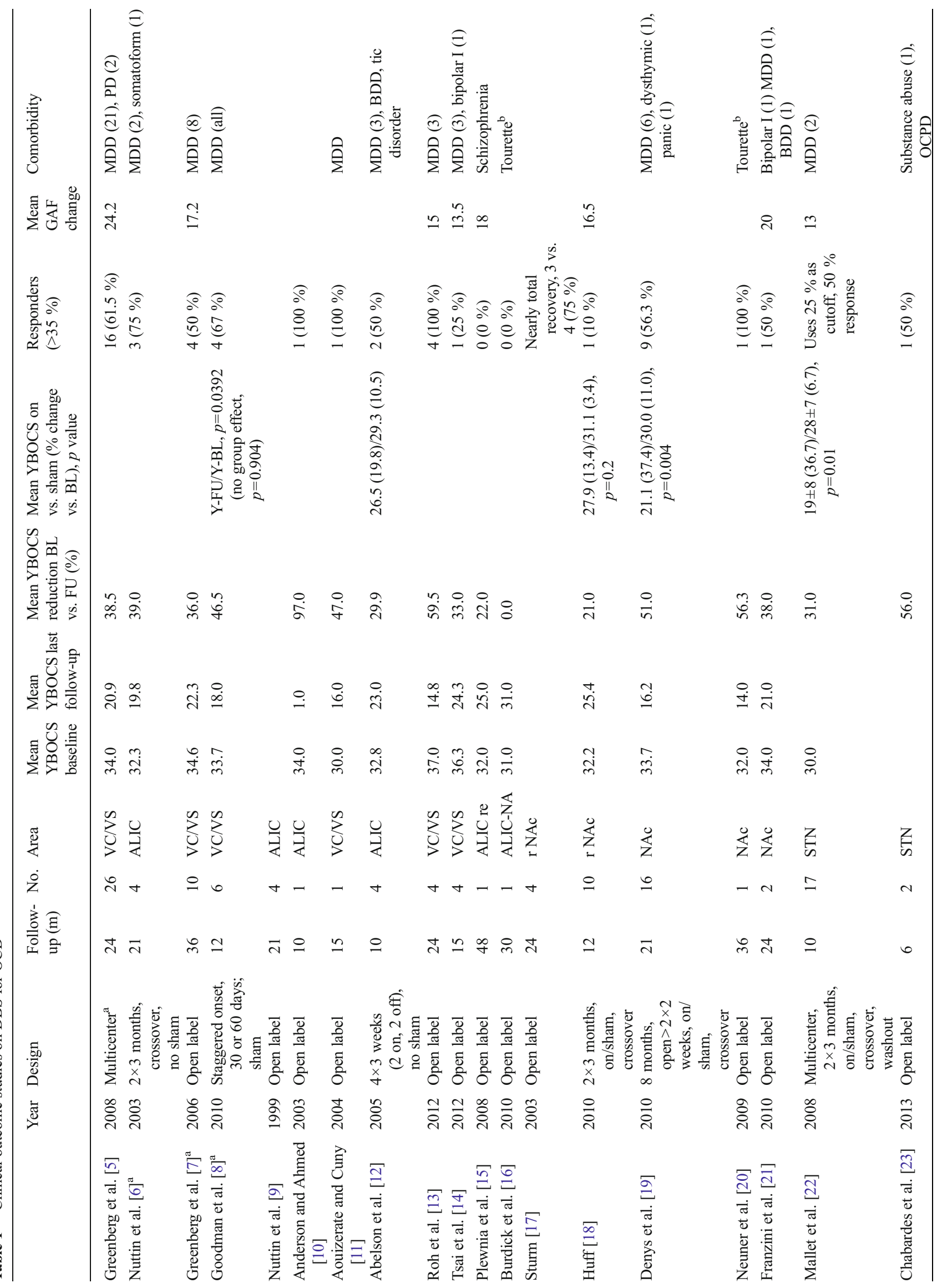




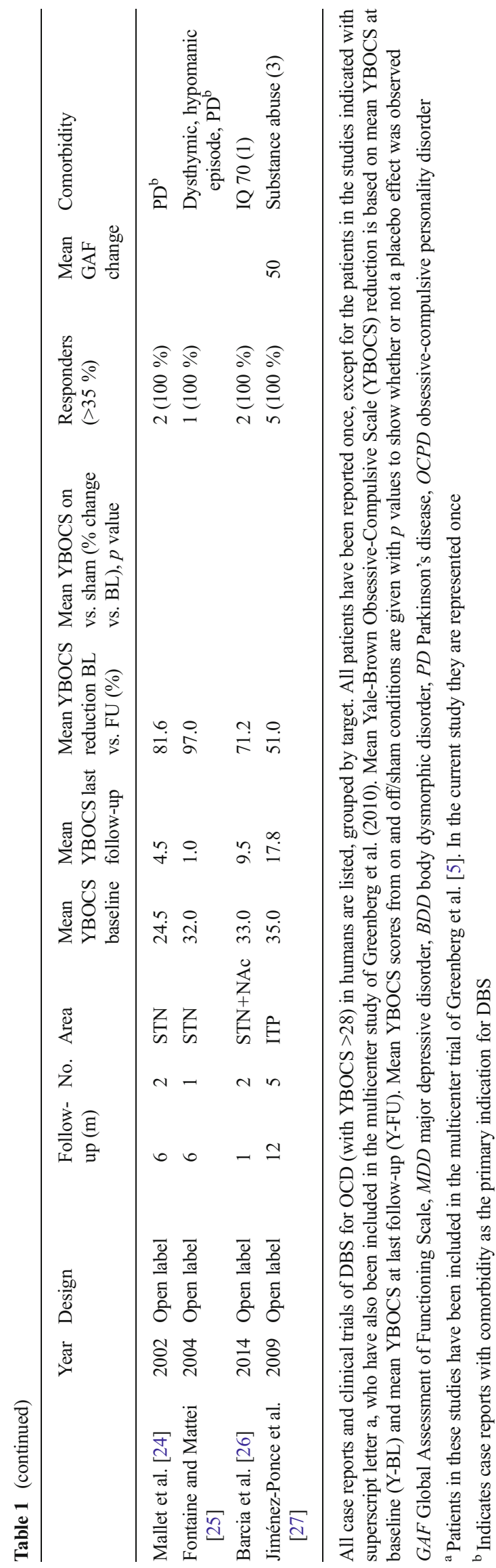

informational signals is crucial. Tellingly, there has been a shift in focus from local effects towards connectivity on the system level [33]. We review clinical and preclinical evidence published in the last 4 years and show that the mechanisms of action of DBS are a combination of excitatory and inhibitory as well as local and distal effects.

\section{Behavioral Studies}

Using selective SRI (SSRI)-validated animal models [34, 35], pathognomonic features of $\mathrm{OCD}$, including cognitive and behavioral inflexibility, attenuated reward processing, and anxiety levels have been assessed in relation to DBS. High-frequency (presumably inhibitory) DBS of the lateral orbitofrontal cortex (OFC) resulted in behavioral inflexibility during a reversal learning task [36], suggesting that local high-frequency stimulation disrupted OFC control over striatal areas, thereby inducing perseverative behaviors. Furthermore, reduced compulsive lever pressing was found with high but not with low-frequency stimulation of the rat's equivalent of the globus pallidus region, which may be explained by activation of passing corticofugal fibers of the internal capsule [37]. Besides these effects on compulsive behaviors, DBS of several other subcortical areas affected unconditioned or conditioned anxiety. Caudate nucleus (CN)-DBS reduced both forms of anxiety, VS-DBS reduced only conditioned anxiety, while NAc and bed nucleus of the stria terminalis (BNST)-DBS did not have any effect on anxiety [38]. Adding to this, VC/VS-DBS during extinction training reduced fear expression and increased fear extinction, which was associated with an increase in OFC, medial prefrontal cortex (mPFC), and amygdala of activity-associated plasticity markers, such as pERK, c-Fos, and BDNF [39•, 40•]. These findings confirm the idea that DBS and CBT, of which extinction is a central element, act as two complementary treatments. Indeed, a recent open-label and uncontrolled clinical DBS trial revealed that the mean YBOCS score and the mean response percentage (minimum $35 \%$ YBOCS reduction) improved from 25 to $42 \%$ and from 50 to $72 \%$, respectively, after augmentation with 24 weeks of CBT [41]. DBS might reduce anxiety through modulation of the stress hormones, shown by an increase in plasma glucose levels in an animal study [42] and YBOCS-correlated normalization of hypercortisolism in humans [43]. In sum, DBS animal models suggest that the stimulation of OFC striatal fibers improves a compulsive behavior, with more ventral stimulation being related to reduced fear conditioning and improved fear extinction as well.

Neurotransmitter Studies

OCD phenomenology, and in particular aberrant reward processing, has been related to dysfunction of dopaminergic neurotransmission [44]. A SPECT study in humans with NAcDBS found a reduced dopamine $\mathrm{D}_{2 / 3}$ receptor-binding 
potential, which was correlated with long-term clinical improvement and increased plasma levels of homovanillic acid, a dopamine metabolite [45]. This indicates DBS-induced increase in striatal dopamine, which might imply that previous findings of increased dopamine transporter (DAT) availability and decreased dopamine $\mathrm{D}_{1}$, a $\mathrm{D}_{2 / 3}$ receptor density in the striatum of OCD patients [46, 47], did not so much indicate downregulation but intrinsic compensatory dopamine release, which is enhanced by DBS. These effects of DBS on dopamine levels might be indirect. A robust finding is that serotonine reuptake inhibitors (SRIs) are effective in reducing symptoms [48] and influence striatal dopamine levels. Possibly, the serotonergic deficits that are typically found in OCD patients, including decreased SERT availability in thalamic and brain stem regions and increased cortical postsynaptic $5-\mathrm{HT}_{2 \mathrm{~A}}$ receptor availability [46, 47], mediate striatal dopaminergic dysfunction. Recent microdialysis studies in healthy rats with NAc-DBS show a rapid increase of both dopamine and serotonin release in frontal cortex areas (OFC and mPFC) [49], but not in the striatal area (NAc) where the stimulating electrode was located [50]. From local recordings in acute rat brain slices, we know that high-frequency stimulation $(140 \mathrm{~Hz})$ of the NAc significantly suppresses spontaneous local neuronal firing. This suppression could be selectively reversed through the application of $\mathrm{GABA}_{\mathrm{B}}$ and non-GABA $\mathrm{A}_{\mathrm{A}}$ antagonists [51]. Possibly, DBS activates GABA-ergic inhibitory interneurons.

\section{Imaging Studies}

Structural brain imaging of OCD patients reveals increased gray matter volume of striatal areas (CN and putamen) and decreased volume of frontal cortical areas (anterior cingulate (ACC) and OFC) [52]. Functional imaging indicates hyperactivity in both striatal and frontal areas (CN and OFC) in resting state and during symptom provocation [53]. Taken together, OCD pathophysiology implies hyperconnectivity between frontal and striatal areas. This long-standing hypothesis was confirmed by DBS-induced normalization of excessive coupling between frontal cortex and NAc in a recent fMRI study in OCD patients. This was paralleled by a normalization of decreased NAc activity [54•]. Recent studies on clinical cohorts of OCD patients treated with STN-DBS [55] and NAc-DBS [54•] show a similar YBOCS-correlated decrease of activity in frontal and striatal areas as had been observed with treatment with SSRIs or CBT [56]. Hyperconnectivity of brain areas in OCD pathophysiology likely includes hyperactivity or hypoactivity in the limbic system, thalamus, and parietal cortex, too [57, 58]. Recent PET findings of DBS-eligible OCD patients include increased resting state metabolism in ACC, occipital cortex, and posterior cerebellum. Several months of $\mathrm{VC} /$ VS-DBS resulted in decreased metabolism in anterior cingulate and prefrontal and orbitofrontal cortices although uncorrelated to YBOCS improvement [59]. Preclinical findings from NAc-
DBS in healthy pigs include activation of prefrontal, insular, cingulate, and parahippocampal regions [60]. Together with the above-discussed evidence from behavioral and neurotransmitter studies, these functional imaging findings converge with the hypothesis that DBS improves OCD by restoring corticostriatal function, possibly in a top-down direction through activation of distant neural assemblies [61, 62].

\section{Electrophysiological Studies}

In line with the aforementioned imaging findings, neuronal populations in the striatum of OCD patients show aberrant discharge patterns. Studies on local recordings in the STN through unconnected DBS electrodes in OCD found increased burst firing in STN neurons in resting state. During symptom provocation, synchrony in the discharge pattern of STN neurons is significantly increased [64]. Patients with lower interburst intervals and higher intraburst frequency had the best YBOCS outcome [65]. Moreover, OCD symptom severity could be predicted by STN discharge patterns. The most severe cases were related to a higher intraburst frequency and more low-frequency oscillations where present [65]. The functional role of this aberrant bursting pattern in OCD, for instance its relation to dopamine levels, remains unclear. To our knowledge, no studies combined in vivo local recordings with active STN-DBS or did local recordings in patients after successful DBS treatment. NAc activity has predictive capacity on a short timescale and has been studied under active stimulation. Intraoperative local recordings in OCD and MDD patients during a gambling task revealed that seconds before their decision is physically manifested, NAc activity already significantly predicts whether subjects will bet high or low. If the result is unexpected, NAc activity successively potentiates when positive and attenuates when negative, which might implicate that the NAc, together with the midbrain dopaminergic system, facilitates reinforcement learning [63]. Although imaging studies point that DBS directly modulates this activity remains unclear, as NAc-DBS in acute rat brain slices had no effect on the discharge pattern or synchrony of local NAc neurons [51], and with NAc-DBS, the bursting pattern of dopaminergic neurons in nigrostriatal and mesolimbic areas in healthy anesthetized rats appeared to be unaltered [66]. One study connected direct local recordings of NAc neurons with surface EEG of the frontal cortex and found top-down-directed synchrony from the frontal cortex to NAc in low-frequency bands during reward anticipation in OCD patients [67]. Moreover, low-frequency oscillations are reduced by NAc-DBS, as was shown by EEG recordings both during resting state [68] and symptom provocation [54•]. These strands of evidence support the hypothesis that the hyperconnectivity of frontal and striatal brain areas, observed with functional imaging, is mediated by low-frequency oscillations, which are typically associated with long distance communication in the brain [69]. 


\section{Conclusion}

Clinical outcome of deep brain stimulation (DBS) for obsessivecompulsive disorder (OCD) shows robust effects in terms of a mean Yale-Brown Obsessive-Compulsive Scale (YBOCS) reduction of $47.7 \%$ and a mean response percentage (minimum $35 \%$ YBOCS reduction) of $58.2 \%$. Additionally, it appears that most patients regain a normal quality of life after DBS. The interpretation of these findings is limited by variations in study designs with respect to the use of a blinded control condition, brain targets, end points, comorbidity, and parameter setting optimization.

Considering the current evidence, it is most likely that the mechanisms of action of DBS are a combination of excitatory and inhibitory as well as local and distal effects. Evidence from DBS animal models converges with human DBS EEG and imaging findings, in that DBS may be effective for OCD by reduction of frontostriatal hyperconnectivity, which is likely achieved through reduction of top-down-directed synchrony and reduction of frontal low-frequency oscillations. DBS appears to counteract striatal dysfunction through an increase in striatal dopamine and through improvement of reward processing. DBS affects anxiety levels through reduction of stress hormones and improvement of fear extinction. The latter relates to epigenetic changes in frontal cortical areas. Further research needs to elucidate how this relates to DBS-induced increase in frontal dopamine and serotonin levels. The observed distal effects with NAc-DBS, in terms of a decrease in prefrontal cortex metabolism, are also observed in DBS of the STN, which is also connected to frontal cortical areas. The functional role of aberrant bursting patterns of STN neurons in OCD, for instance its relation to dopamine levels, remains unclear. Future studies could combine in vivo local recordings with active STN-DBS or perform local recordings in patients after successful DBS treatment.

Acknowledgments ER gratefully acknowledges the support he obtained from the Netherlands Organization for Scientific Research (NWO) in the form of a VIDI grant.

\section{Compliance with Ethics Guidelines}

Conflict of Interest Damiaan Denys works as a consultant for Lundbeck and received honoraria from Medtronic and Lundbeck, royalties from UpToDate, and travel accommodations from Medtronic and Lundbeck. Martijn Figee, Erik Rietveld, and Maarten van Westen have no relevant disclosures to report.

Human and Animal Rights and Informed Consent This article does not contain any studies with human or animal subjects performed by the author.

Open Access This article is distributed under the terms of the Creative Commons Attribution License which permits any use, distribution, and reproduction in any medium, provided the original author(s) and the source are credited.

\section{References}

Papers of particular interest, published recently, have been highlighted as:

- Of importance

- Of major importance

1. Ruscio AM, Stein DJ, Chiu WT, Kessler RC. The epidemiology of obsessive-compulsive disorder in the National Comorbidity Survey Replication. Mol Psychiatry. 2008;15:53-63.

2. Pallanti $\mathrm{S}$ et al. Treatment non-response in OCD: methodological issues and operational definitions. Int J Neuropsychopharmacol. 2002;5:181-91.

3.• Greenberg BD, Rauch SL, Haber SN. Invasive circuitry-based neurotherapeutics: stereotactic ablation and deep brain stimulation for OCD. Neuropsychopharmacology. 2009;35:317-36. This thorough review can be seen as background of the current article, in which we discuss evidence from the last four years, as it discusses the neurobiology of OCD in great detail and with a broad scope.

4. Höflich A et al. Neuropsychiatric deep brain stimulation for translational neuroimaging. Neuroimage. 2013;79:30-41.

5. Greenberg BD et al. Deep brain stimulation of the ventral internal capsule/ventral striatum for obsessive-compulsive disorder: worldwide experience. Mol Psychiatry. 2008;15:64-79.

6. Nuttin B, Gabriels LA, Cosyns PR, Meyerson BA. Long-term electrical capsular stimulation in patients with obsessive-compulsive disorder. Neurosurgery. 2003;52:1263-74.

7. Greenberg BD et al. Three-year outcomes in deep brain stimulation for highly resistant obsessive-compulsive disorder. Neuropsychopharmacology. 2006;31:2384-93.

8. Goodman WK et al. Deep brain stimulation for intractable obsessive compulsive disorder: pilot study using a blinded, staggeredonset design. Biol Psychiatry. 2010;67:535-42.

9. Nuttin B, Cosyns P, Demeulemeester H, Gybels J. Electrical stimulation in anterior limbs of internal capsules in patients with obsessive-compulsive disorder. Lancet. 1999;354:1526.

10. Anderson D, Ahmed A. Treatment of patients with intractable obsessive-compulsive disorder with anterior capsular stimulation. J Neurosurg. 2003;98:1104-8.

11. Aouizerate B, Cuny E. Deep brain stimulation of the ventral caudate nucleus in the treatment of obsessive-compulsive disorder and major depression. J Neurosurg. 2004;101:682-6.

12. Abelson JL et al. Deep brain stimulation for refractory obsessivecompulsive disorder. Biol Psychiatry. 2005;57:510-6.

13. Roh D, Seok W, Woo J, Kim C. Long-term follow-up of deep brain stimulation for refractory obsessive-compulsive disorder. Psychiatry Res. 2012;200:1067-70.

14. Tsai H-C et al. Pilot study of deep brain stimulation in refractory obsessive-compulsive disorder ethnic Chinese patients. Psychiatry Clin Neurosci. 2012;66:303-12.

15. Plewnia C, Schober F, Rilk A. Sustained improvement of obsessive-compulsive disorder by deep brain stimulation in a woman with residual schizophrenia. Int J Neuropsychopharmacol. 2008;11: 1181-3.

16. Burdick A et al. Lack of benefit of accumbens/capsular deep brain stimulation in a patient with both tics and obsessive-compulsive disorder. Neurocase. 2010;16:37-41.

17. Sturm V et al. The nucleus accumbens: a target for deep brain stimulation in obsessive-compulsive- and anxiety-disorders. J Chem Neuroanat. 2003;26:293-9.

18. Huff $\mathrm{W}$ et al. Unilateral deep brain stimulation of the nucleus accumbens in patients with treatment-resistant obsessive-compulsive 
disorder: outcomes after one year. Clin Neurol Neurosurg. 2010;112:137-43.

19. Denys D et al. Deep brain stimulation of the nucleus accumbens for treatment-refractory obsessive-compulsive disorder. Arch Gen Psychiatry. 2010;67:1061-8.

20. Neuner I, Podoll K, Lenartz D, Sturm V, Schneider F. Deep brain stimulation in the nucleus accumbens for intractable Tourette's syndrome: follow-up report of 36 months. Biol Psychiatry. 2009;65:5-6.

21. Franzini A et al. Deep-brain stimulation of the nucleus accumbens in obsessive compulsive disorder: clinical, surgical and electrophysiological considerations in two consecutive patients. Neurol Sci. 2010;31:353-9.

22. Mallet $\mathrm{L}$ et al. Subthalamic nucleus stimulation in severe obsessive-compulsive disorder. N Engl J Med. 2008;359:2121-34.

23. Chabardès $\mathrm{S}$ et al. Deep brain stimulation for obsessive-compulsive disorder: subthalamic nucleus target. World Neurosurg. 2013;80: S31.e1-8.

24. Mallet L et al. Compulsions, Parkinson's disease, and stimulation. Lancet. 2002;360:1302-4

25. Fontaine D, Mattei V. Effect of subthalamic nucleus stimulation on obsessive-compulsive disorder in a patient with Parkinson disease. J Neurosurg. 2004;100:1084-6.

26. Barcia JA, Arza R, Saceda J. Deep brain stimulation for obsessivecompulsive disorder: is the side relevant? Stereotact Funct Neurosurg. 2014;92:31-6.

27. Jiménez-Ponce F, Velasco-Campos F, Castro-Farfán G. Preliminary study in patients with obsessive-compulsive disorder treated with electrical stimulation in the inferior thalamic peduncle. Neurosurgery. 2009;65:203-9.

28. De Koning PP, Figee M, van den Munckhof P, Schuurman PR, Denys D. Current status of deep brain stimulation for obsessivecompulsive disorder: a clinical review of different targets. Curr Psychiatry Rep. 2011;13:274-82.

29. Nangunoori R, Tomycz ND, Quigley M, Oh MY, Whiting DM. Deep brain stimulation for psychiatric diseases: a pooled analysis of published studies employing disease-specific standardized outcome scales. Stereotact Funct Neurosurg. 2013;91:345-54.

30. Figee M, Schuurman R, \& Denys D, Comment on Nangunoori, et al. Deep brain stimulation for psychiatric diseases: a pooled analysis of published studies employing disease-specific standardized outcome scales. Stereotact Funct Neurosurg. Forthcoming.

31. Ooms P et al. Deep brain stimulation for obsessive-compulsive disorders: long-term analysis of quality of life. J Neurol Neurosurg Psychiatry. 2014;85:153-8.

32. Ooms $\mathrm{P}$ et al. Rebound of affective symptoms following acute cessation of deep brain stimulation in obsessive-compulsive disorder. Brain Stimul. 2014;7:727-31.

33. Montgomery EB, Gale JT. Mechanisms of action of deep brain stimulation (DBS). Neurosci Biobehav Rev. 2008;32:388-407.

34. Albelda N, Joel D. Animal models of obsessive-compulsive disorder: exploring pharmacology and neural substrates. Neurosci Biobehav Rev. 2012;36:47-63.

35. Camilla d'Angelo L-S et al. Animal models of obsessivecompulsive spectrum disorders. CNS Spectr. 2014;19:28-49.

36. Klanker M, Post G, Joosten R, Feenstra M, Denys D. Deep brain stimulation in the lateral orbitofrontal cortex impairs spatial reversal learning. Behav Brain Res. 2013;245:7-12.

37. Klavir O, Winter C, Joel D. High but not low frequency stimulation of both the globus pallidus and the entopeduncular nucleus reduces "compulsive" lever-pressing in rats. Behav Brain Res. 2011;216: 84-93.

38. Van Dijk A et al. Deep brain stimulation affects conditioned and unconditioned anxiety in different brain areas. Transl Psychiatry. 2013;3:e289.
39. Rodriguez-Romaguera J, Do Monte FHM, Quirk GJ. Deep brain stimulation of the ventral striatum enhances extinction of conditioned fear. Proc Natl Acad Sci U S A. 2012;109:1-6.

40. Do-Monte FH, Rodriguez-Romaguera J, Rosas-Vidal LE, Quirk GJ. Deep brain stimulation of the ventral striatum increases BDNF in the fear extinction circuit. Front Behav Neurosci. 2013;7:102. Both studies of Do-Monte and Rodriguez-Romaguera correlate epigenetic changes with behavioral data.

41. Mantione M, Nieman D, Figee M, Denys D. Cognitive-behavioral therapy augments the effects of deep brain stimulation in obsessivecompulsive disorder. Psychol Med. 2014;44(16):3515-22.

42. Diepenbroek $\mathrm{C}$ et al. Alterations in blood glucose and plasma glucagon concentrations during deep brain stimulation in the shell region of the nucleus accumbens in rats. Front Neurosci. 2013;7:1-8

43. De Koning PP et al. Deep brain stimulation for obsessivecompulsive disorder is associated with cortisol changes. Psychoneuroendocrinology. 2013;38:1455-9.

44. Figee $\mathrm{M}$ et al. Dysfunctional reward circuitry in obsessivecompulsive disorder. Biol Psychiatry. 2011;69:867-74.

45. Figee $\mathrm{M}$ et al. Deep brain stimulation induces striatal dopamine release in obsessive-compulsive disorder. Biol Psychiatry. 2014;75:647-52.

46. Nikolaus S, Antke C, Beu M, Müller H, Nikolaus S. Cortical GABA, striatal dopamine and midbrain serotonin as the key players in compulsive and anxiety disorders - results from in vivo imaging studies. Rev Neurosci. 2010;139:119-39.

47. Zohar J, Greenberg B, \& Denys D. Obsessive-compulsive disorder. Handbook of clinical neurology. Elsevier B.V.; 2012. 106:375-90.

48. Denys D. Pharmacotherapy of obsessive-compulsive disorder and obsessive-compulsive spectrum disorders. Psychiatr Clin North Am. 2006;29:553-84.

49. Van Dijk A, Klompmakers AA, Feenstra MGP, Denys D. Deep brain stimulation of the accumbens increases dopamine, serotonin, and noradrenaline in the prefrontal cortex. J Neurochem. 2012;123: 897-903.

50. Van Dijk A, Mason O, Klompmakers AA, Feenstra MGP, Denys D. Unilateral deep brain stimulation in the nucleus accumbens core does not affect local monoamine release. J Neurosci Methods. 2011;202:113-8.

51. Xie Y, et al. High-frequency electrical stimulation suppresses cholinergic accumbens interneurons in acute rat brain slices through GABA B receptors. Eur J Neurosci. 2014;1-10. doi: 10.1111/ejn. 12736.

52. Menzies L et al. Integrating evidence from neuroimaging and neuropsychological studies of obsessive-compulsive disorder: the orbitofrontostriatal model revisited. Neurosci Biobehav Rev. 2008;32:525-49.

53. Rauch SL, Dougherty D, Malone DA, Rezai AR. A functional neuroimaging investigation of deep brain stimulation in patients with obsessive-compulsive disorder. J Neurosurg. 2006;104: 558-65.

54.• Figee $\mathrm{M}$ et al. Deep brain stimulation restores frontostriatal network activity in obsessive-compulsive disorder. Nat Neurosci. 2013;16: 386-7. This study provides a strong body of evidence for frontostriatal hyperconnectivity underlying OCD symptomatology and shows how EEG might explain imaging findings.

55. Le Jeune F et al. Decrease of prefrontal metabolism after subthalamic stimulation in obsessive-compulsive disorder: a positron emission tomography study. Biol Psychiatry. 2010;68:1016-22.

56. Saxena S, Rauch SL. Functional neuroimaging and the neuroanatomy of obsessive-compulsive disorder. Psychiatr Clin North Am. 2000;57:26-35.

57. Kopell BH, Greenberg B, Rezai AR. Deep brain stimulation for psychiatric disorders. J Clin Neurophysiol. 2004;21:51-67. 
58. Rotge J et al. Anatomical alterations and symptom-related functional activity in obsessive-compulsive disorder are correlated in the lateral orbitofrontal cortex. Biol Psychiatry. 2010;67:e37-8.

59. Suetens K, Nuttin B, Gabriëls L, Van Laere K. Differences in metabolic network modulation between capsulotomy and deep-brain stimulation for refractory obsessive-compulsive disorder. J Nucl Med. 2014;55:951-9.

60. Knight EJ et al. Nucleus accumbens deep brain stimulation results in insula and prefrontal activation: a large animal FMRI study. PLoS One. 2013;8:e56640.

61. Montgomery EB. Deep brain stimulation programming. Oxford University Press; 2010.

62. Grill WM, Cantrell MB, Robertson MS. Antidromic propagation of action potentials in branched axons: implications for the mechanisms of action of deep brain stimulation. J Comput Neurosci. 2008;24:81-93.

63. Patel SR et al. Single-neuron responses in the human nucleus accumbens during a financial decision-making task. J Neurosci. 2012;32:7311-5.
64. Bastin J, et al. Changes of oscillatory activity in the subthalamic nucleus during obsessive-compulsive disorder symptoms: two case reports. Cortex. 2014;2-7. doi: 10.1016/j.cortex.2013.12.007.

65. Welter M-L et al. Basal ganglia dysfunction in OCD: subthalamic neuronal activity correlates with symptoms severity and predicts high-frequency stimulation efficacy. Transl Psychiatry. 2011;1:e5.

66. Sesia T, Bizup B, Grace A. Nucleus accumbens high-frequency stimulation selectively impacts nigrostriatal dopaminergic neurons. Int J Neuropsychopharmacol. 2014;17:421-7.

67. Cohen MX et al. Top-down-directed synchrony from medial frontal cortex to nucleus accumbens during reward anticipation. Hum Brain Mapp. 2012;33:246-52.

68. Smolders R et al. Deep brain stimulation targeted at the nucleus accumbens decreases the potential for pathologic network communication. Biol Psychiatry. 2013;74:e27-8.

69. Varela F, Lachaux J, Rodriguez E, Martinerie J. The brainweb: phase synchronization and large-scale integration. Nat Rev Neurosci. 2001;2:229-39. 